\title{
Design and Implementation of 2-Input OR Gate based on XGM properties of Semiconductor Optical Amplifier
}

\author{
Chandra Kamal Borgohain ${ }^{1}$, Chakresh Kumar ${ }^{2}$ and Satyajit Bora ${ }^{1}$
}

\begin{abstract}
In this paper we have implemented an alloptical OR-gate using simultaneous Four-Wave Mixing (FWM) and Cross-Gain Modulation (XGM) in a semiconductor optical amplifier (SOA). Which incorporates two semiconductor optical amplifiers (SOAs). The proposed OR logic is numerically simulated by solving nonlinear coupled equations that explain cross gain modulation (XGM) effect in individual SOAs.
\end{abstract}

Keywords - Semiconductor Optical Amplifier, Optical Logic Gates, Cross Gain Modulation, SOA, Four Wave Mixing.

\section{INTRODUCTION}

At present, SOAs are the prime candidates for use in advanced optical communication functional components. The growth of large scale integration of SOA technology offers economical, high performance devices. SOAs exhibit non-linear properties due to carrier density changes induced by differences in power of the input signal. While these non-linear properties create problems for the use of SOAs as simple linear gain elements, they can be exploited to perform functions that are typically carried out by electronic signal processing circuits. In the case of all applications mentioned above, the data signal is processed in optical form, rather than first being converted to an electrical signal. SOA-based devices are compact, stable, integration-capable, and potentially independent of polarization and wavelength [4]. Also, it has the advantages of low switching energy and low latency [5]. A computation that might take more than eleven years by the conventional electronic takes only one single hour [6].The use of the optical fibers as nonlinear elements in switching devices gives so high speed operation since the nonlinear response of the fiber is extremely fast and the possibility of creating a rectangular switching by using a dispersive walk-off between the data and

${ }^{1}$ Department of Electronics and Communication Engineering, Tezpur (central) University, INDIA

${ }^{2}$ University School of Information \& Communication Technology, Guru Gobind Singh Indraprastha University, Dwarka, New Delhi110078, India

E-mail-borgohainchandrakama1176@gmail.com the control pulse but the nonlinear coefficient is usually very small in conventional fibers[8]. In this paper the full logic is developed in MATLAB platform.

\section{SIMULATION METHOD}

In this approach, we have taken the reference equations from Ref. [15] and different parameters which are taken into consideration are tabulated below in Table I. It is assumed that input pump, and probe pulses have the same temporal width as well as perfect pulse overlap, and in all of the cases, their powers are set to a ratio of 10:1. Numerical simulations have been undertaken to investigate the amplification of strong picoseconds optical pulses in semiconductor optical amplifiers (SOAs), taking into account carrier heating, spectral whole burning, carrier-carrier scattering (CCS) and carrier photon scattering (CPS). The result of interference of two co polarized pulses when propagating into SOA, one pump pulse at central frequency $\omega 1$ and the other probe pulse at central frequency $\omega 0$, induce a bit of carrier density pulsation at the frequency detuning $\Omega$ $=\omega 1-\omega 0$. This results a generation of a new frequency pulse at $\omega 2=\omega 0-\Omega=2 \omega 0-\omega 1$. The new pulse is phase conjugate replica of the probe pulses, and can be extracted from the input pulses using an optical filter. Here, $A_{j}(Z, t), j=0,1,2$, correspond to the slowly varying envelopes of the pump, the probe, and the conjugate pulses, respectively, and $\Omega=\omega 1$ $\omega 0$, is the frequency detuning.

The following Equation has been taken into consideration

$A_{0}(L, t)=A_{0}(0, t) e^{\left(\frac{1}{2}\right)(1-i \alpha) h}$

Where, $A_{0}(0, t)$, is the input pump pulse amplitude at any end of SOA,

$A_{0}(L, t)$, is the input pump pulse amplitude at any length $L$ of SOA, 
$L$ is the length of SOA, $t$ is time. The rest of parameters are already defined in Table I.

$$
\begin{aligned}
& A_{1}(L, t)=A_{0}(0, t)\left[e^{\left(\frac{1}{2}\right)(1-i \alpha) h-\eta_{10}\left|A_{0}(0, t)\right|^{2}\left(e^{h}-1\right)}\right] \cosh \\
& {\left[\left(\frac{1}{2}\right) \sqrt{\eta_{02} \eta_{01}^{*}}\left|A_{0}(0, t)\right|^{2}\left(e^{h}-1\right)\right]}
\end{aligned}
$$

Where, $A_{1}(0, t)$, is the input probe pulse amplitude at any end of SOA.

$A_{1}(L, t)$, is the input probe pulse amplitude at any length $L$ of SOA.

$L=$ length of SOA. $\mathrm{t}$ is time. The rest of parameters are already defined in Table I.

$$
\begin{aligned}
& A_{2}(L, t)=\frac{A_{1}^{*}(L, t) A_{0}(L, t)}{A_{0}^{*}(L, t)} \sqrt{\frac{\eta_{01}}{\eta_{02}^{*}}} x \\
& \sinh \left[\left(\frac{1}{2}\right) \sqrt{\eta_{01} \eta_{02}^{*}}\left|A_{0}(L, t)\right|^{2} e^{-h}\left(e^{h}-1\right)\right]
\end{aligned}
$$

\section{Where}

$A_{2}(0, t)$ is input conjugate pulse amplitude at any end of SOA.

$A_{2}(L, t)$ is input conjugate pulse amplitude at any length $L$ of SOA. $L=$ length of SOA, $t$ is time. Rest parameters are already defined in Table I.

$\eta_{01}=\eta_{01}^{C D}+\eta_{01}^{C H}+\eta_{01}^{S H B}$

Where,

$\eta_{01}^{C D}=\varepsilon_{c d} \frac{1-i \alpha}{\left(\left(1+i \Omega \tau_{1}\right)+\left(1+i \Omega \tau_{S}\right)\right)}$

$\eta_{01}^{C H}=\varepsilon_{t} \frac{1-i \alpha_{T}}{\left(\left(1+i \Omega \tau_{h}\right)+\left(1+i \Omega \tau_{1}\right)\right)}$

$\eta_{01}^{S H B}=\varepsilon_{S h b} \frac{1-i \alpha_{s h b}}{1+i \Omega \tau_{1}}$

The amplification function $h$ and coupling coefficient Пij are defined in [15]. The effects of carrier depletion, carrier heating, spectral hole burning, twophoton absorption, and ultrafast nonlinear refraction are taken into account, leading to a successful description of wave mixing for optical pulses with strong pulse energy, and/or with pulse widths larger than several hundred femto seconds, as well as in active or passive optical waveguides. The parameters $\alpha, \tau$, and $\varepsilon$ determine the strength and nature of the wave mixing process caused by each of the intraband mechanisms and their relative significance. The values of $\alpha$, tand $\varepsilon$ cannot be determined unanimously.
TABLE I

PARAMETERS USED IN SIMULATION WORK

\begin{tabular}{lccc}
\hline \multicolumn{1}{c}{ Parameters } & Symbols & Values & Unit \\
\hline Length of the amplifier & $\mathrm{L}$ & 450 & $\mu \mathrm{m}$ \\
$\begin{array}{l}\text { Small signal gain } \\
\text { Carrier lifetime }\end{array}$ & $\mathrm{G}$ & $1.54 * 10^{-4}$ & $\mathrm{~m}^{-1}$ \\
$\begin{array}{l}\text { Nonlinear gain } \\
\text { compression for carrier } \\
\text { heating }\end{array}$ & $\tau_{\mathrm{s}}$ & 300 & $\mathrm{ps}$ \\
$\begin{array}{l}\text { Nonlinear gain } \\
\text { compression for spectral } \\
\text { hole burning }\end{array}$ & $\varepsilon_{\mathrm{t} h \mathrm{~b}}$ & 0.13 & $\mathrm{w}^{-1}$ \\
$\begin{array}{l}\text { Traditional line width } \\
\text { enhancement factor }\end{array}$ & $\alpha$ & 0.07 & \\
$\begin{array}{l}\text { Temperature l line width } \\
\text { enhancement factor }\end{array}$ & $\alpha_{\mathrm{T}}$ & 5.0 & \\
$\begin{array}{l}\text { Line width enhancement } \\
\text { factor for spectral hole } \\
\text { burning }\end{array}$ & & 3.0 & \\
$\begin{array}{l}\text { Time for carrier-carrier } \\
\text { scattering }\end{array}$ & $\alpha_{\mathrm{shb}}$ & 0.1 & \\
$\begin{array}{l}\text { Time for carrier photon } \\
\text { scattering }\end{array}$ & $\tau_{1}$ & 50 & $\mathrm{fs}$ \\
$\begin{array}{l}\text { Carrier depletion } \\
\text { coefficient }\end{array}$ & $\tau_{\mathrm{h}}$ & 700 & $\mathrm{fs}$ \\
\hline \hline
\end{tabular}

III. RESULTS AND DISCUSSION

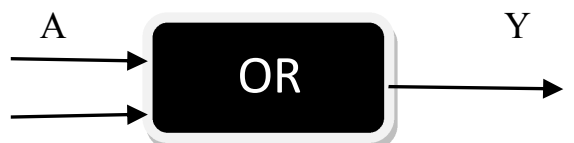

B

Fig. 1. OR Gate

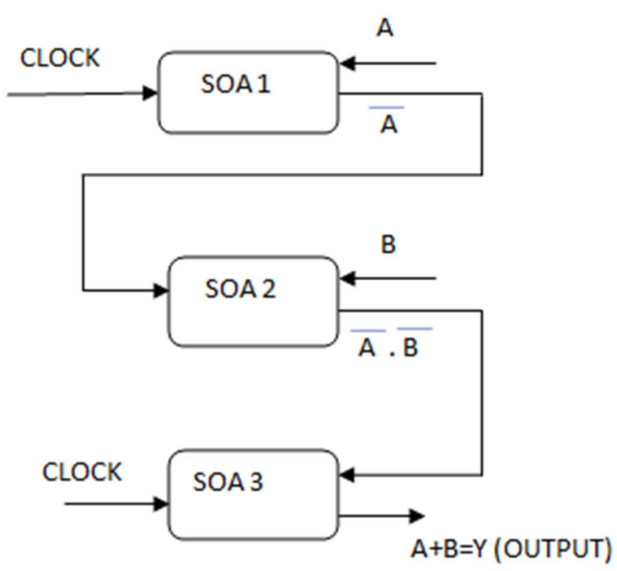

Fig. 2. OR using SOAs 
TABLE II

TRUTH TABLE FOR OR LOGIC CAN BE GIVEN AS

\begin{tabular}{ccc}
\hline \hline $\mathrm{A}$ & $\mathrm{B}$ & $\mathrm{A}+\mathrm{B}=\mathrm{Y}$ \\
\hline 0 & 0 & 0 \\
0 & 1 & 1 \\
1 & 0 & 1 \\
1 & 1 & 1 \\
\hline \hline
\end{tabular}

Fig. 2 shows the OR gate using SOAs. In SOA1 'A' acts as an input pump pulse and 'CLOCK' signal acts as an input probe pulse. In SOA 2 the output of SOA1 acts as input probe pulse and B acts as an input pump pulse. Similarly, in SOA 3 CLOCK signal acts as input probe pulse and the output of SOA2 acts as input pump pulse, which gives the required Boolean expression $\mathrm{A}+\mathrm{B}$.

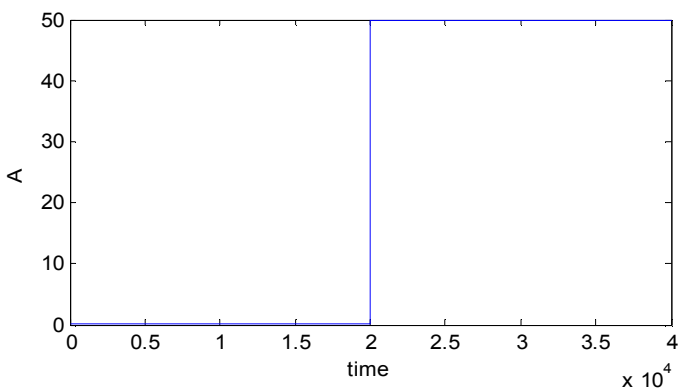

Fig. 3. Waveform of $A=\left[\begin{array}{llll}0 & 0 & 1 & 1\end{array}\right]$

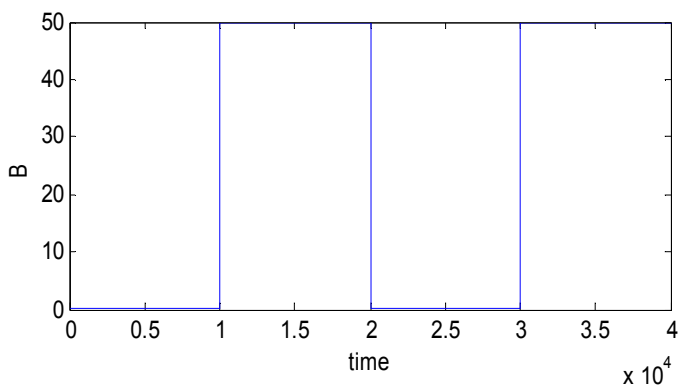

Fig. 4. Waveform of $\mathrm{B}=\left[\begin{array}{llll}0 & 1 & 0 & 1\end{array}\right]$

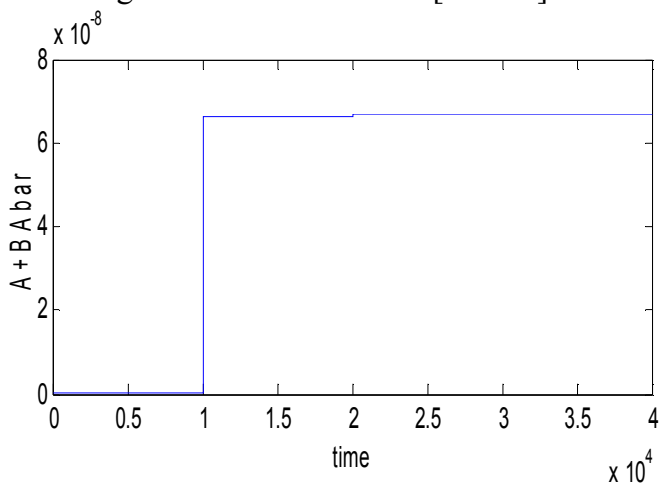

Fig. 5. Waveform of OR output $=\left[\begin{array}{llll}0 & 1 & 1 & 1\end{array}\right]$

From Fig. 3, 4 and 5 we can observe that the simulation results clearly verify the truth table of OR gate.

\section{CONCLUSIONS}

We have proposed and implemented a new all-optical OR gate using analytic solutions of nonlinear effects in semiconductor optical amplifier. This research has guided readers to design all-optical OR logic circuits so that anyone can construct any types of all-optical different logic circuits by utilizing the detailed process of designing the OR Gate. The simulated result verifies the truth table.

\section{REFERENCES}

[1] Jae Hun Kim, Young Il Kim, Young Tae Byun,* Young Min Jhon,Seok Lee, Sun Ho Kim and Deok HaWoo,Photonics Research Center, "All Optical Logic Gates Using Semiconductor Optical-Amplifier-Based Devices and Their Applications", Korea Institute of Science and Technology, Seoul 136791

[2] T. Fjelde, D.Wolfson, A. Kloch, B. Dagens, A. Coquelin,I. Guillemot, F. Gaborit, F. Poingt and M. Renaud, Electron.Lett. 36, 1863 (2000).

[3] K. Vlachos, K. Zoiros, T. Houbavlis, A. Hatziefremidis and H. Avramopoulos, Proc. LEOS 768, 2 (1999).

[4] Alistair J. Poustie, K. J. Blow, A. E. Kelly and R. J. Manning, "All-optical parity checker". BT Laboratories, Martlesham Heath, Ipswich, IP5 3RE, U.K.,Tel: +44 1473 646442 Fax: +44 1473646885

[5] C.K.Yow, Y.J.Chai, K.A.Williams, RV.Penty, 1.H.White. "Enhanced performance of an all-optical parity checker using a single Mach-Zehnder interferometer". Centre for Photonics Communications, Cambridge University Engineering Department, Trumpington Street, Cambridge CP2 1PZ United Kingdom Telephone: +4401223 740103Fmc: 1-84 01223 740102E-mail cky21@cam ac uk

[6] Hossin Abdeldayem et al., " Recent Advances in Photonic Devices forOptical Computing", NASA Marshall Space Flight Center, Space Sciences laboratory, Huntsville, al 35812

[7] B. E. A. Saleh, and M. C. Teich, Fundamentals of Photonics (JohnWiley \& Sons, New York, 1991), Chap. 19.

[8] Bengt- Erik Olsson, "All Optical Switching with the Nonlinear Optical Loop Mirror", School of electrical and computer engineering, Chalniers University of technology, Goteborg, Sweden, Technical Report No. 330,1998.

[9] Xia, T.J., Liang, Y., Ahn, K.H.: 'All-optical packet-drop demonstration using $100 \mathrm{~Gb} / \mathrm{s}$ words by integrating fiberbased components', IEEE photonic technology letters, 1998, 10, (1), pp.153-155.

[10] Bogoni, A., Poti, L., Proietti, R., Meloni, G., Ponzini, F., Ghelfi, P.: 'Regenerative and reconfigurable all-optical logic gates for ultra-fast applications', Electronics Letters, 2005, 41, (7), pp.435-436.

[11] Stubkjaer, K.E.: 'Semiconductor optical amplifier-based all optical gates for high-speed optical processing', IEEE Journal on selected topics in quantum electronics, 2000, 6, (6), pp.1428-1435.IEEE photonic technology letters,2001, 13, (7), pp. 750-752.

[12] Martinez, J.M., Ramos, F., Marti, J.: 'All-optical packet headerprocessor based on cascaded SOA-MZIs', Electronics letters, 2004,40,(14), pp.894-895.

[13] Kim, J., Jhon, Y, Byun, Y., Lee, S., Woo, D., Kim, S.: ‘Alloptical XOR gate using semiconductor optical amplifiers without additional input beam', IEEE photonic technology letters, 2002, 14, (10), pp.1436-1438.

[14] Jae Hun Kim, Young Min Jhon, Young Tae Byun, Seok Lee, Deok HaWoo, and Sun Ho Kim "All-Optical XOR Gate 
Using Semiconductor Optical Amplifiers Without Additional Input Beam “,IEEE PHOTONICSTECHNOLOGY LETTERS, VOL. 14, NO. 10, OCTOBER 2002

[15] S Junqlang et al., "Analytical solution of Four wave mixing between picosecond optical pulses in semiconductor optical amplifiers with cross gain modulation and probe depletion", Microwave and Optical technology Letters, Vol. 28, No. 1, pp $78-82,2001$.

[16] Vikrant k Srivastava, Devendra Chack, Vishnu Priye, "2Input AND Gate For Fast Gating and Switching Based On XGM Properties of InGaAsP Semiconductor Optical Amplifier", International Journal of Advanced Computer Science and Applications, Vol. 1, No. 5, (2010).

[17] Vikrant k Srivastava, Devendra Chack, Vishnu Priye, Chakresh Kumar. "All-Optical XOR Gate Based on XGM Properties of SOA". International Conference on Computational Intelligence and Communication Network, IEEE computer society, 2010. 